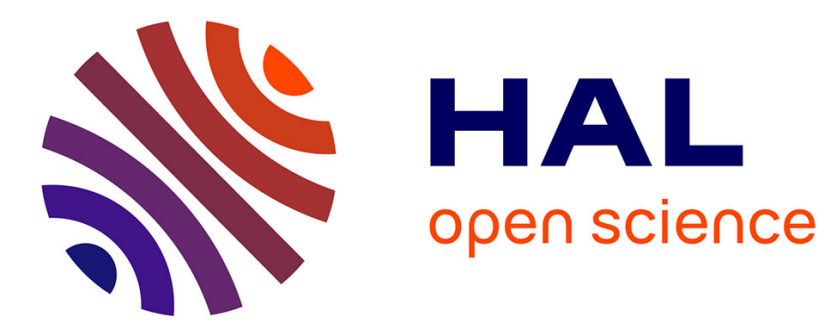

\title{
IDENTIFICATION DE PRÉCIPITÉS DE NITRURE D'ALUMINIUM DANS DES ÉCHANTILLONS DE FER
}

\author{
A. Fourdeux, Jean Sévely, G. Zanchi, Yolande Kihn
}

\section{- To cite this version:}

A. Fourdeux, Jean Sévely, G. Zanchi, Yolande Kihn. IDENTIFICATION DE PRÉCIPITÉS DE NITRURE D'ALUMINIUM DANS DES ÉCHANTILLONS DE FER. Journal de Physique Colloques, 1984, 45 (C2), pp.C2-691-C2-694. 10.1051/jphyscol:19842160 . jpa-00223833

\section{HAL Id: jpa-00223833 https://hal.science/jpa-00223833}

Submitted on 1 Jan 1984

HAL is a multi-disciplinary open access archive for the deposit and dissemination of scientific research documents, whether they are published or not. The documents may come from teaching and research institutions in France or abroad, or from public or private research centers.
L'archive ouverte pluridisciplinaire HAL, est destinée au dépôt et à la diffusion de documents scientifiques de niveau recherche, publiés ou non, émanant des établissements d'enseignement et de recherche français ou étrangers, des laboratoires publics ou privés. 
JOURNAL DE PHYSIQUE

Colloque C2, supplément au n², Tome 45, février 1984

page $C 2-691$

IDENTIFICATION DE PRÉCIPITÉS DE NITRURE D'ALUMINIUM DANS DES ÉCHANTILLONS DE FER

\author{
A. Fourdeux, J. Sevely*, G. Zanchi ${ }^{*}$ et Y. Kihn ${ }^{*}$ \\ Ecole des Mines de Saint-Etienne, 158 cours Fauriel, \\ 42023 Saint-Etienne Cedex, France \\ * Laboratoire d'optique Electronique du CNRS, 29 me Jeanne Marvig, \\ 31055 Toulouse Cedex, France
}

\begin{abstract}
Résumé : La spectroscopie de pertes d'énergie d'électrons à haute tension (1 MV) a êté utilisée pour confirmer la composition chimique de fins précipités de nitrure d'aluminium qui apparaissent dans les aciers faiblement alliés. La technique a permis de bien mettre en évidence un enrichissement en aluminium des zones contenant les précipités. Elle confirme que ceux-ci sont consitués de nitrure d'aluminium.
\end{abstract}

Abstract : Electron Energy Loss Spectroscopy at high voltage (1 MV) has been used to establish the chemical composition of thin precipitates observed in low-carbon steel samples which show a poor ductibility at high temperature, $750-1100^{\circ} \mathrm{C}$. The technique has allowed to confirm that the precipitates correspond to Al $\mathrm{N}$ formation.

Les aciers faiblement alliés, à bas carbone, calmés à l'aluminium, présentent, en général, une baisse de ductilité à chaud. Ce phénomène se manifeste dans un domaine de tempèratures compris entre $750^{\circ} \mathrm{C}$ et $1100^{\circ} \mathrm{C}$. Il a été attribué à une précipitation fine de nitrure d'aluminium : Al N. Aucune preuve convaincante n'a été apportée à cette explication, en particulier l'identification de nitrure d'aluminium par microscopie et diffraction électroniques a étê sujette à de nombreuses controverses. II apparait donc important de compléter ces résultats par une analyse chimique locale maintenant possible par spectroscopie de pertes d'énergie d'électrons. Des expériences effectuées avec cette technique par Egerton et al (1) ont apporté un premier élément de réponse et confirmé l'existence d'une forte concentration en azote dans ces types de prēcipitës mais les spectres ne mettaient pas en évidence la présence d'aluminium.

Les expériences ont été reprises en spectroscopie de pertes d'énergie d'êlectrons à haute tension (1 MV) dont on peut attendre une plus grande sensibilité dans la mise en évidence des signaux caractéristiques d'excitation de niveaux atomiques profonds et l'étude d'échantilions plus épais (2).

\title{
I - ETUDE DES ECHANTILLONS EN MICROSCOPIE ELECTRONIQUE CLASSIQUE
}

Dans les alliages industriels, 1'identification des précipités est difficile en raison de la présence, mal contrôlée, d'oligo-êlêments autres que l'azote et l'aluminium. Pour contourner cette difficulté, un alliage (Fe, $\mathrm{N}, \mathrm{Al}$ ) contenant $500 \mathrm{ppm}$ d'aluminium et $310 \mathrm{ppm}$ d'azote a étê élaborè à partir de fer de haute pureté préparé à l'Ecole des Mines de Saint-Etienne (3). Sa teneur en oligo-éléments est en ppm la suivante : $C<10,0<20, S<1, M n<1$ et $\mathrm{Si}<10$. L'alliage a été maintenu à $1000^{\circ} \mathrm{C}$ pendant 10 heures puis refroidi lentement afin d'obtenir de nombreux préciptēs de taille convenable pour permettre leur identification en microscopie et diffraction électroniques classiques.

Des prēcipitês de forme géométrique et de taille $<500 \mathrm{~nm}$ ont été observés sur lames minces par microscopie électronique en transmission à $100 \mathrm{kV}$, (Fig.1). Leur identification par diffraction électronique a été tentée. Elle amenait à conclure à la formation de nitrures d'aluminium, Al $N,(4)$. 


\section{II - ANALYSE EN SPECTROMETRIE DE PERTES D'ENERGIE D'ELECTRONS}

Les échantilions ont été ensuite observés à l'aide du microscope électronique 1,2 MV équipé d'un système d'analyse en énergie des électrons de type $\Omega(5)$. Ce dispositif permet soit de former le spectre des pertes d'énergie correspondant à une aire sélectionnée de l'objet, soit de former une image filtrée de l'ëchantilion. C'est-à-dire, une image obtenue en sélectionnant les électrons correspondant à des pertes d'énergie choisies.

\section{a) Le spectre des pertes d'énergie}

L'objet est examiné avec des électrons de 1 MV. Le spectre des pertes d'énergie enregistré est associé à une aire sélectionnée de l'objet de $1000 \AA$ de diamètre environ. Cette aire est dêlimitêe par un diaphragme de sélection de $10 \mu \mathrm{m}$ de diamètre disposé dans le plan image de la lēre lentille intermédiaire du microscope. La por-tion de l'échantilion qui est analysée est directement choisie sur l'image. Les pertes d'énergie qui sont mises en évidence pour ces expériences sont comprises entre quelques eV et $2500 \mathrm{eV}$. L'analyse en énergie des électrons associés à l'image des précipités, (Fig. 2), fait apparaitre sur le spectre, (Fig. 3 A), des distributions caractéristiques dont les seuils sont situés à 400 , 530,730 et $1540 \mathrm{eV}$. Ces distributions sont respectivement attribuées à l'excitation des niveaux atomiques profonds $K$ de 1 'azote, $K$ de 1 'oxygène, $L_{23}$ du fer et $K$ de 1 'aluminium. L'analyse sur une portion voisine de l'objet ne contenant pas le précipité, (Fig. 3B), ne permet de mettre en évidence que les distributions caractéristiques $K$ de 7 'oxygène à $530 \mathrm{eV}$ et L23 du fer à $730 \mathrm{eV}$. La comparaison de tels spectres permet de conclure à la présence d'azote et d'aluminium dans les précipités et de confirmer que ceux-ci correspondent à la formation de nitrure d'aluminium dans la matrice qui est essentiellement constituée de fer. La concentration en azote et en aluminium, respectivement 310 et 500 ppm, y est trop faible pour que ces éléments puissent être dëtectés par la méthode. Il en est de même des oligo-éléments qui ne sont présents qu'en concentrations de T'ordre du ppm.

b) Filtrage d'image sur pertes caractëristiques

La fenêtre d'énergie choisie pour la formation de l'image peut être positionnée sur la distribution caractéristique d'excitation d'un niveau atomique profond d'un élément déterminé. L'image obtenue permet de dresser une carte de la distribution de cet élément dans 1'objet. La résolution de cette image est peu modifiée par rapport à celle de 1 'image normale (5). Cette méthode présente donc un grand intérêt pour étudier la répartition des éléments majeurs dans un échantillon.

La concentration d'azote dans les précipités a pu àinsiêtre confirmée par la comparaison d'images filtrées sur les diverses pertes de part et d'autre du seuil de Ta distribution caractéristique $K$ de cet élément, (Fig. 4). Ainsi, le contraste de l'image du précipité par rapport à la matrice est multiplié par un facteur de l'ordre de 3 lorsque 1 'on compare les images filtrées, ( $4 \mathrm{~A}$ et $4 \mathrm{~B}$ ), respectivement sur $7 \mathrm{a}$ fenêtre d'énergie $\Delta E=375 \pm 10 \mathrm{eV}$, dans le fond continu du spectre juste avant le

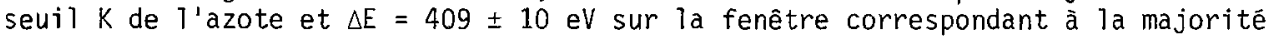
des électrons ayant excité le niveau $K$ de l'azote.

La distribution associée à l'aluminium était de trop faible intensité pour pouvoir être exploitêe de la même manière.

Comme le montre cet exemple, la technique de spectroscopie de pertes d'énergie d'électrons permet de détecter la présence d'éléments, même légers et en faible concentration dans un échantilion. La localisation du précipité dans la matrice ne permet pas de développer d'analyse quantitative précise. La sensibilité de la méthode est de l'ordre de quelques pour cent. Elle dépend de la nature des éléments recherchés. Sa résolution spaciale dépend beaucoup de celle du microscope électronique. Dans le dispositif que nous avons utilisé, elle est définie par la taille du diaphragme de sêlection et se trouve limitée à $50 \mathrm{~nm}$. 


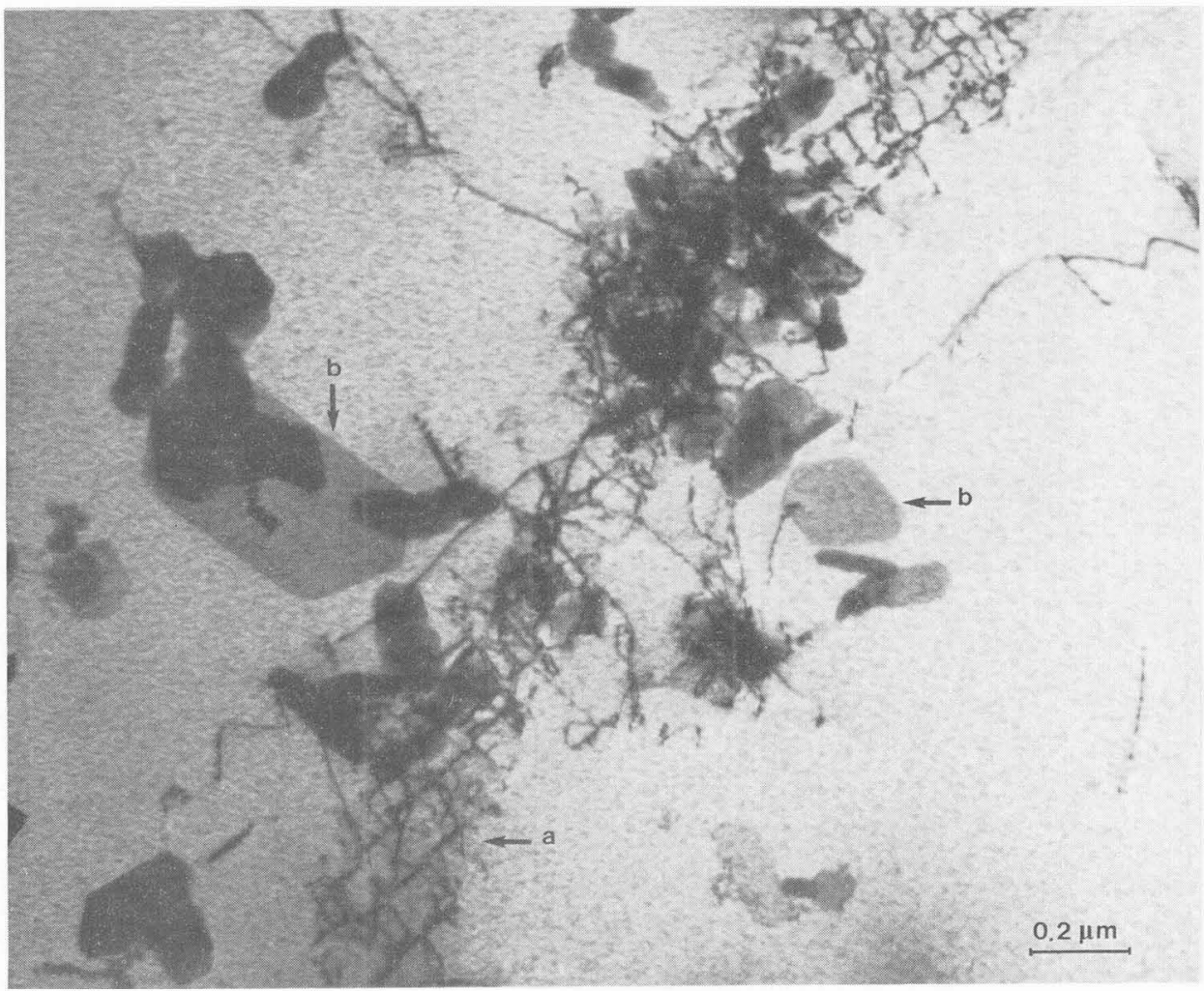

Fig. 1 : Image en fond clair à $100 \mathrm{kV}$ de lames minces d'acier à bas carbone mise en évidence de sous-joints (a) et de précipités (b).

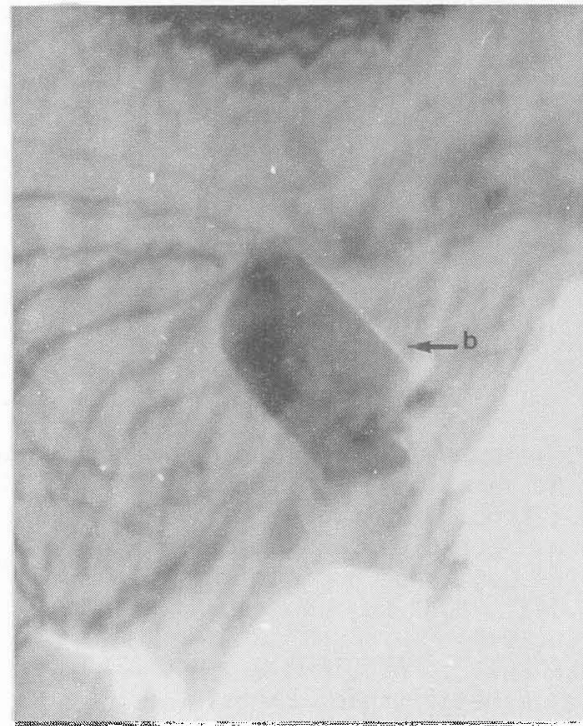

Fig. 2 : Image en fond clair à $1 M V$ de la plage analysée - mise en évidence du précipitê (b).
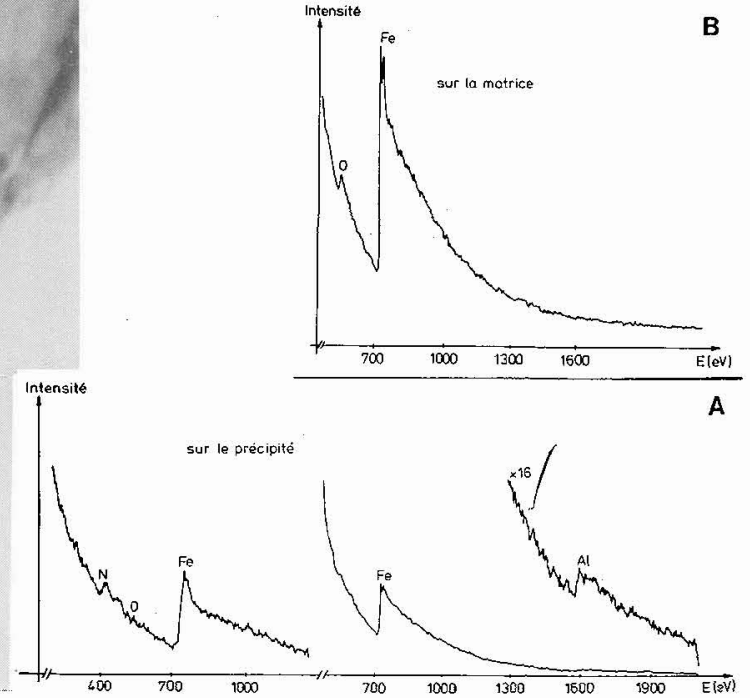

Fig. 3 : Spectres de pertes d'énergie, sur le précipité (A) sur la matrice (B) 

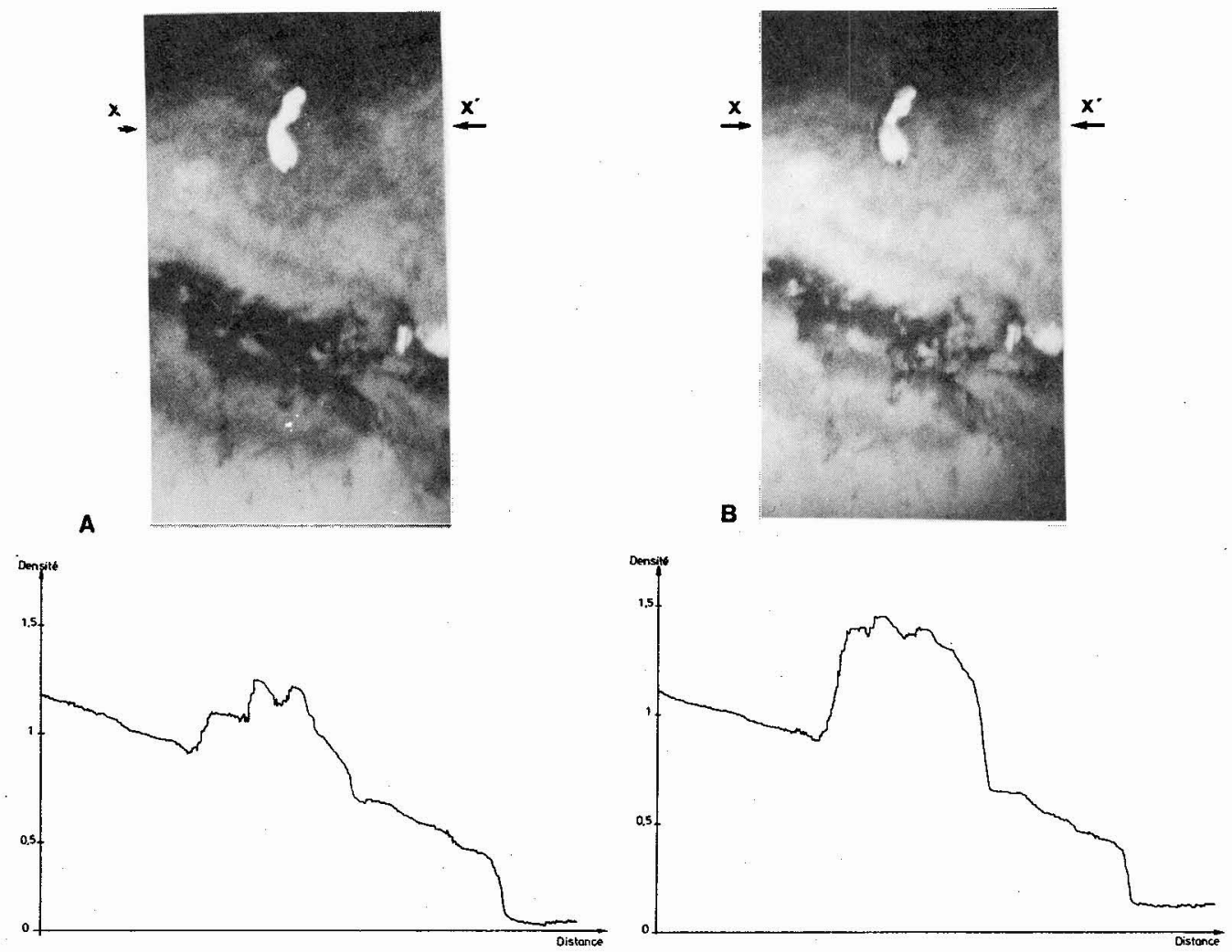

Fig. 4 : Comparaison d'images filtrées du précipité et enregistrement microdensitométriques associés suivant 1 a direction $x x^{\prime}$

$A$ - image avant le seuil $K$ de l'azote

$B$ - image immëdiatement après le seuil $K$ de l'azote.

\section{REFERENCES}

(1) EGERTON R.F., ROSSOUW C.J., WHELAN M.J., Developments in Electron Microscopy and Analysis (1976) (Academic Press, ed. by J.A. Venables), (1976) 129.

(2) ZANCHI G., KIHN Y., SEVELY J., JOUFFREY B., Proc. 7 th International Conference on High Voltage Electron Microscopy, Lawrence Berkeley Laboratory, (1983), 85. also SEVELY J., KIHN Y., ZANCHI G., JOUFFREY B., these proceedings.

(3) TARDY R., CHERBLANC G., ROCHETTE J., BOOS J.Y., GOUX C., Journées d'automne de la Société Française de Métallurgie, Paris, (1975).

(4) GOYARD T., FOURDEUX A., KOBYLANSKI A., PALENGAT R., BEGUINOT J., BLONDEAU R., Journées d'automne de la Sociétê Française de Métallurgie, Paris, (1979).

(5) ZANCHI G., SEVELY J., JOUFFREY B., J. Microsc. Spectrosc. Electronique, 2 (1977) 95. 